\title{
High-Frequency Modeling of Star-Connected Induction Motors Using Multilayer Perceptron
}

This paper was downloaded from TechRxiv (https://www.techrxiv.org).

\section{LICENSE}

CC BY 4.0

SUBMISSION DATE / POSTED DATE

$10-02-2022$ / 11-02-2022

\section{CITATION}

Zhao, Zhenyu; Fan, Fei; Sun, Quqin; Tu, Pengfei; See, Kye Yak (2022): High-Frequency Modeling of StarConnected Induction Motors Using Multilayer Perceptron. TechRxiv. Preprint. https://doi.org/10.36227/techrxiv.19152572.v1

$\mathrm{DOI}$

10.36227/techrxiv.19152572.v1 


\title{
High-Frequency Modeling of Star-Connected Induction Motors Using Multilayer Perceptron
}

\author{
Zhenyu Zhao, Member, IEEE, Fei Fan, Member IEEE, Quqin Sun, \\ Pengfei Tu, Member, IEEE, and Kye Yak See, Senior Member, IEEE
}

\begin{abstract}
This letter presents an easy-to-use accurate high-frequency (HF) behavioral model of star-connected induction motors with a simple and straightforward parameterization process for conducted electromagnetic interference (EMI) analysis. The HF model contains nine circuit elements per phase with a simple structure. These circuit elements are determined using a multilayer perceptron (MLP) neural network, where only the individual phase-to-frame impedance is needed for training purposes. Compared with parameterization methods in conventional motor modeling, the MLP significantly simplifies the parameterization process. Using two different induction motors (1.5 hp and $7.5 \mathrm{hp}$ ) as case studies, experiments verify the capability and accuracy of the HF model to predict the motor common-mode (CM) and differential-mode (DM) impedances in the $\mathrm{HF}$ range of $150 \mathrm{kHz}$ to $30 \mathrm{MHz}$.
\end{abstract}

Index Terms-Electromagnetic interference (EMI), highfrequency (HF) modeling, induction motor, multilayer perceptron (MLP), parameterization.

\section{INTRODUCTION}

$\mathrm{V}$ ARIABLE frequency drive (VFD) systems have been widely used in many industrial applications because of their excellent power conversion efficiency [1]. With the advancement in power semiconductor technology, higher switching frequency has become norms in the designs of VFD systems, which also generates higher electromagnetic interference (EMI) due to fast edge rates [2]. EMI modeling and simulation serves as an effective means to predict system EMI behavior and optimize EMI mitigation strategies at the design stage before final product implementation. With the increasing switching frequencies in VFD systems, an accurate and reliable high-frequency (HF) model of induction motors is necessary to ensure good EMI prediction results [3]. This paper focuses on the $\mathrm{HF}$ range of $150 \mathrm{kHz}-30 \mathrm{MHz}$, which is the mandatory requirements by many EMI standards [4]-[6].

Conventionally, the HF models of induction motors are categorized into two main types, the finite element analysis (FEA)-based models and the behavioral models [7]. FEA-based models are created via 2-D or 3-D electromagnetic analysis using numerical methods [8]-[10]. Behavioral models are developed through impedance measurements and curve fitting techniques [3], [7], [11]-[14]. Numerous behavioral models have been proposed to represent the motor's common-mode $(\mathrm{CM})$ and differential-mode (DM) impedance characteristics for conducted EMI analysis. High-accuracy behavioral models

This research work was supported by Nanyang Technological University. (Corresponding author: Fei Fan.)

The authors are with the School of Electrical and Electronics Engineering, Nanyang Technological University, Singapore. (fanf0003@e.ntu.edu.sg).

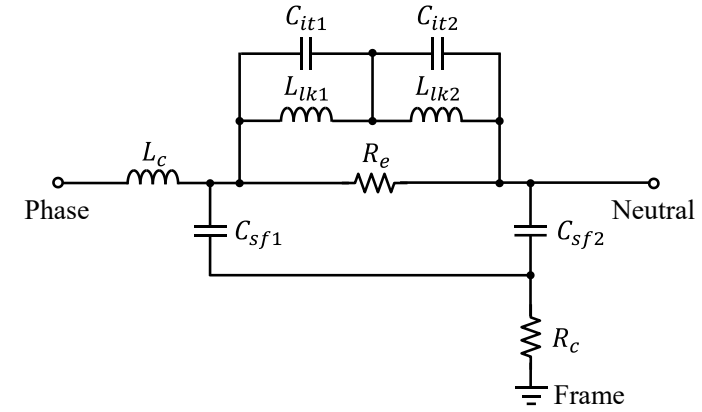

Fig. 1. Proposed HF model of star-connected induction motor per phase.

usually require more circuit elements and complex parameterization process, which sacrifices model simplicity and practicability [7].

This letter proposes a simplified and accurate HF behavioral model of star-connected induction motors with a simple and straightforward parameterization process. The model consists of a simplified circuit with nine circuit elements per phase. These circuit elements can be easily determined using a multilayer perceptron (MLP) neural network, where only the individual phase-to-frame impedance is needed for training purposes. Compared with the parameterization methods in conventional motor modeling, the MLP simplifies the parameterization process. Among various neural networks, although the MLP is less competent in multi-scale feature extraction than more advanced deep neural networks, it is still preferred in this application for its adequate regression ability with less computational cost.

In this letter, two different induction motors (1.5 hp and 7.5 hp) are selected as case studies. Section II describes the proposed HF modeling and parameterization process. Section III demonstrates its accuracy to predict the motor CM and DM impedances in the HF range of $150 \mathrm{kHz}$ to $30 \mathrm{MHz}$. Finally, Section IV concludes this letter.

\section{HF MODELING OF MOTOR USING MLP}

Fig. 1 shows the proposed HF model of star-connected induction motor per phase, which consists of nine physically meaningful circuit elements. $L_{c}$ represents the combined inductance of the stator winding leakage inductance in the first few turns and the inductance of internal feed conductors connected to the stator winding. $C_{s f 1}$ and $C_{s f 2}$ account for the stator-to-frame capacitances. $R_{c}$ denotes the combined resistance of the HF stator copper skin effect resistance and the frame resistance. $L_{l k 1}$ and $L_{l k 2}$ represent the stator winding leakage inductances. $C_{i t 1}$ and $C_{i t 2}$ account for the stator winding interturn capacitances. $R_{e}$ denotes the HF stator eddy- 


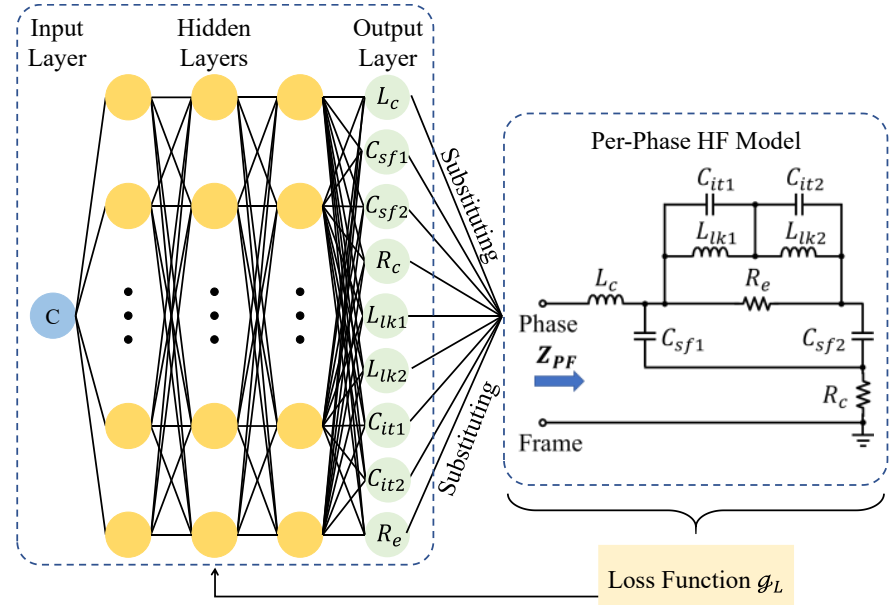

Fig. 2. Parameterization process using a MLP neural network.

current losses. For the three-phase HF model, it is constructed using three per-phase HF models shown in Fig. 1 based on the motor winding connection. Due to manufacturing tolerances and layout differences [8], there may be slight differences in the values of these circuit elements for the three phases, namely phases $\mathrm{A}, \mathrm{B}$, and $\mathrm{C}$.

To determine the values of these circuit elements of each phase, a MLP neural network is employed, where Fig. 2 shows the parametrization process. As illustrated in Fig. 2, the MLP neural network consists of five layers, namely one input layer, three hidden layers, and one output layer. The input of the MLP is a random constant value $\mathrm{C}$, and the output of the MLP are the nine circuit elements (i.e., $L_{c}, C_{s f 1}, C_{s f 2}, R_{c}, L_{l k 1}, L_{l k 2}, C_{i t 1}$, $C_{i t 2}$, and $R_{e}$ ). The units of $L_{c}, R_{c}$, and $R_{e}$ are set as microhenry $(\mu \mathrm{H})$, ohm $(\Omega)$, and kiloohm $(\mathrm{k} \Omega)$, respectively; the units of $C_{s f 1}, C_{s f 2}, C_{i t 1}$, and $C_{i t 2}$ are set as nanofarad (nF); the units of $L_{l k 1}$ and $L_{l k 2}$ are set as millihenry $(\mathrm{mH})$ [7], [12]. The number of neurons in the three hidden layers are set as 100, 500, and 100 , respectively. The loss function $\left(g_{L}\right)$ is defined in terms of the motor's phase-to-frame impedance as follows:

$$
g_{L}=\frac{1}{2 m}\left\{\sqrt{\sum_{i=1}^{m}\left(\left|\boldsymbol{Z}_{\boldsymbol{P F}}\right|-\left|\boldsymbol{Z}_{\boldsymbol{P F}}^{\prime}\right|\right)^{2}}+\sqrt{\sum_{i=1}^{m}\left(\angle \boldsymbol{Z}_{\boldsymbol{P F}}-\angle \boldsymbol{Z}_{\boldsymbol{P F}}^{\prime}\right)^{2}}\right\}
$$

where $m$ is the number of frequency points. $\boldsymbol{Z}_{\boldsymbol{P F}}^{\prime}$ represents the reference phase-to-frame impedance measured with an impedance analyzer. When measuring $\boldsymbol{Z}_{\boldsymbol{P F}}^{\prime}$ of one phase, the probe of the impedance analyzer connects between the phase and frame, and the neutral is disconnected. $\boldsymbol{Z}_{\boldsymbol{P F}}$ represents the trained phase-to-frame impedance via the MLP. $\boldsymbol{Z}_{\boldsymbol{P F}}$ is trained through substituting the results of the output layer into the perphase HF model. By minimizing $g_{L}$, the values of these circuit elements per phase can be determined, and finally, the HF model of induction motor can be constructed. It should be noted that $g_{L}$ represents the sum of the impedance magnitude loss and phase loss, and both losses have the same weightage (i.e., 1/2).

For proof of concept, Figs. 3 and 4 show the measured and trained phase-to-frame impedances for phases $\mathrm{A}, \mathrm{B}$, and $\mathrm{C}$ of a $1.5 \mathrm{hp}($ TECO AESV38041R50FUN) and a $7.5 \mathrm{hp}$ (TEOC AEEBKB067R50FM) induction motor, respectively. The
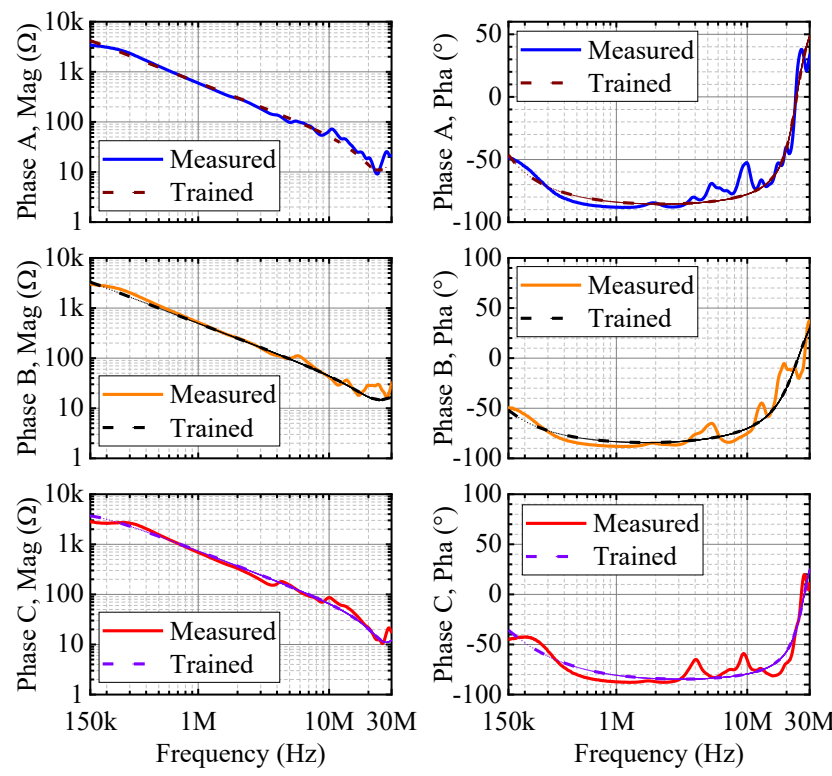

Fig. 3. Measured $\left(\boldsymbol{Z}_{\boldsymbol{P F}}^{\prime}\right)$ and trained $\left(\boldsymbol{Z}_{\boldsymbol{P F}}\right)$ phase-to-frame impedances for phases $\mathrm{A}, \mathrm{B}$, and $\mathrm{C}$ of the $1.5 \mathrm{hp}$ induction motor.
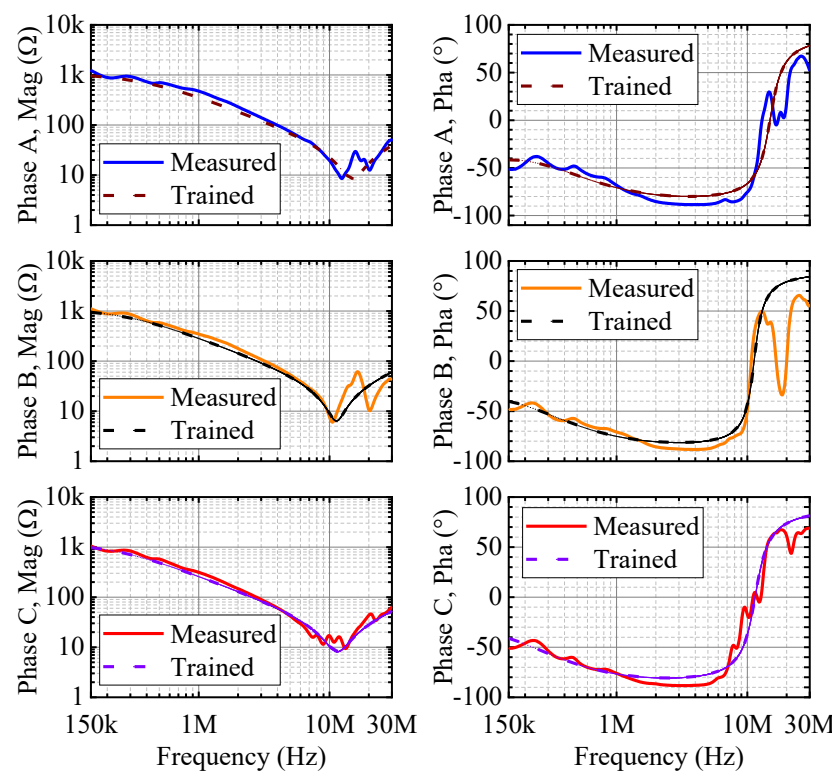

Fig. 4. Measured $\left(\boldsymbol{Z}_{\boldsymbol{P F}}^{\prime}\right)$ and trained $\left(\boldsymbol{Z}_{\boldsymbol{P F}}\right)$ phase-to-frame impedances for phases $\mathrm{A}, \mathrm{B}$, and $\mathrm{C}$ of the $7.5 \mathrm{hp}$ induction motor.

individual phase-to-frame impedance is measured with a Bode 100 impedance analyzer. The iteration number of the training process is 1000. As shown in Figs. 3 and 4, the trained phaseto-frame impedances have demonstrated good agreement with those of the measured ones for all three phases of the induction motors in the $\mathrm{HF}$ range of $150 \mathrm{kHz}-30 \mathrm{MHz}$. Based on the trained phase-to-frame impedances, the values of the circuit elements for the three phases of the induction motors are obtained, as given in Table I. Hence, the motors' HF models are constructed based on these circuit elements.

\section{EXPERIMENTAL VALIDATION}

For experimental validation, the same induction motors (1.5 hp and $7.5 \mathrm{hp}$ ) are selected as case studies. In order to verify the accuracy of the proposed HF model to predict motor $\mathrm{CM}\left(\boldsymbol{Z}_{\boldsymbol{C M}}\right)$ and DM $\left(\boldsymbol{Z}_{\boldsymbol{D} \boldsymbol{M}}\right)$ impedances in the HF range of interest, the 
TABLE I

Determined Circuit Elements for Phases A, B AND C OF THE Two INDUCTION MOTORS (1.5 HP AND $7.5 \mathrm{HP}$ )

\begin{tabular}{lcccccc}
\hline \hline \multirow{2}{*}{ Element } & \multicolumn{3}{c}{$1.5 \mathrm{hp}$ induction motor } & \multicolumn{3}{c}{7.5 hp induction motor } \\
\cline { 2 - 7 } & $\mathrm{A}$ & $\mathrm{B}$ & $\mathrm{C}$ & $\mathrm{A}$ & $\mathrm{B}$ & $\mathrm{C}$ \\
\hline$L_{c}(\mu \mathrm{H})$ & 0.170 & 0.132 & 0.158 & 0.270 & 0.370 & 0.321 \\
$C_{s f 1}(\mathrm{nF})$ & 0.060 & 0.080 & 0.054 & 0.104 & 0.130 & 0.152 \\
$C_{s f 2}(\mathrm{nF})$ & 1.488 & 2.581 & 1.614 & 2.235 & 2.760 & 3.430 \\
$R_{c}(\Omega)$ & 10.358 & 14.524 & 10.890 & 7.807 & 5.828 & 7.737 \\
$L_{l k 1}(\mathrm{mH})$ & 4.547 & 5.808 & 5.771 & 3.991 & 2.913 & 3.878 \\
$L_{l k 2}(\mathrm{mH})$ & 4.547 & 5.808 & 5.771 & 3.991 & 2.913 & 3.878 \\
$C_{i t 1}(\mathrm{nF})$ & 0.466 & 0.533 & 0.360 & 0.743 & 0.960 & 1.030 \\
$C_{i t 2}(\mathrm{nF})$ & 0.466 & 0.533 & 0.360 & 0.743 & 0.960 & 1.030 \\
$R_{e}(\mathrm{k} \Omega)$ & 5.202 & 4.700 & 4.038 & 0.832 & 0.873 & 0.974 \\
\hline \hline
\end{tabular}

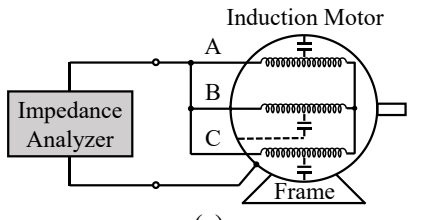

(a)

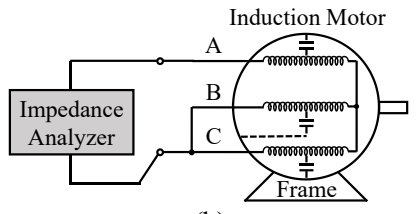

(b)
Fig. 5. Configuration for measuring motor (a) $\boldsymbol{Z}_{\boldsymbol{C M}}$ and (b) $\boldsymbol{Z}_{\boldsymbol{D M}}$

actual measured $\boldsymbol{Z}_{\boldsymbol{C M}}$ and $\boldsymbol{Z}_{\boldsymbol{D M}}$ of the induction motors are used as references. Figs. 5(a) and (b) show the respective configuration for measuring the motor's $\boldsymbol{Z}_{\boldsymbol{C M}}$ and $\boldsymbol{Z}_{\boldsymbol{D M}}$ with a Bode 100 impedance analyzer [7].

Based on the obtained motors' three-phase circuit elements in Table 1, Figs. 6 and 7 show the simulated $\boldsymbol{Z}_{\boldsymbol{C} \boldsymbol{M}}$ and $\boldsymbol{Z}_{\boldsymbol{D} \boldsymbol{M}}$ in the $\mathrm{HF}$ range of $150 \mathrm{kHz}-30 \mathrm{MHz}$ for the $1.5 \mathrm{hp}$ and $7.5 \mathrm{hp}$ induction motors, respectively. In addition, the actual measured $\boldsymbol{Z}_{\boldsymbol{C M}}$ and $\boldsymbol{Z}_{\boldsymbol{D M}}$ of the same induction motors are also shown in the figures for comparison purposes. As seen in Figs. 6 and 7, both magnitude and phase of the simulated $\boldsymbol{Z}_{\boldsymbol{C M}}$ and $\boldsymbol{Z}_{\boldsymbol{D M}}$ for the $1.5 \mathrm{hp}$ induction motor show good agreement with the those of the actual measured from $150 \mathrm{kHz}$ to $30 \mathrm{MHz}$. Although the simulated $\boldsymbol{Z}_{\boldsymbol{C} \boldsymbol{M}}$ and $\boldsymbol{Z}_{\boldsymbol{D M}}$ for the $7.5 \mathrm{hp}$ induction motor show some discrepancy from the measured results in certain frequency regions above $10 \mathrm{MHz}$, they still exhibit reasonably good consistency over most of the HF range from $150 \mathrm{kHz}-30$ $\mathrm{MHz}$. Based on these experimental results, the proposed HF model has shown its capability to predict the motor's CM and DM impedances with good accuracy.

\section{CONCLUSION}

This letter proposes a simplified and accurate HF behavioral model of star-connected induction motors with a simple and straightforward MLP-based parameterization process for conducted EMI analysis. The motor's circuit elements can be determined by measuring individual phase-to-frame impedance for training purposes. It greatly simplifies the parameterization process as compared with the conventional HF modeling methods. Experimental results of two induction motors $(1.5 \mathrm{hp}$ and $7.5 \mathrm{hp}$ ) have demonstrated the accuracy of the proposed HF modeling approach to predict the motor's CM and DM impedances in the $\mathrm{HF}$ range of $150 \mathrm{kHz}-30 \mathrm{MHz}$.

\section{REFERENCES}

[1] H. Soltani, P. Davari, F. Zare, and F. Blaabjerg, "Effects of modulation techniques on the input current interharmonics of adjustable speed drives," IEEE Trans. Ind. Electron., vol. 65, no. 1, pp. 167-178, Jan 2018.
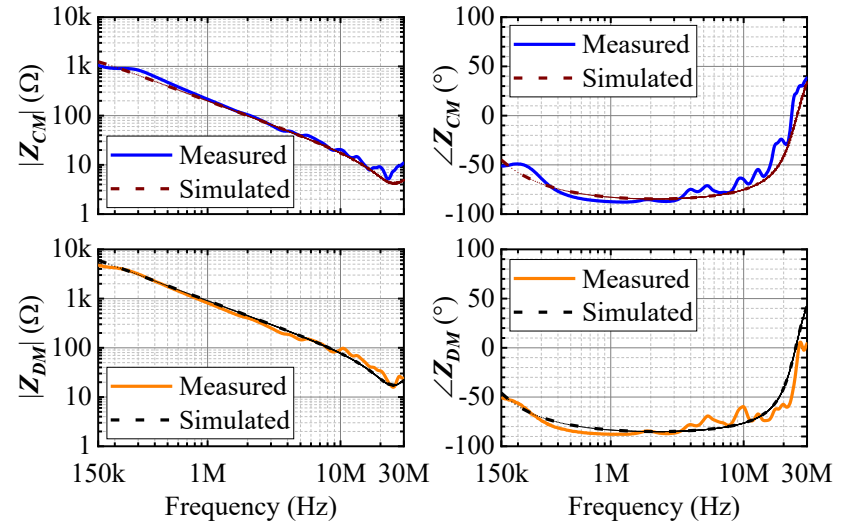

Fig. 6. Measured and simulated $\boldsymbol{Z}_{\boldsymbol{C} \boldsymbol{M}}$ and $\boldsymbol{Z}_{\boldsymbol{D} \boldsymbol{M}}$ of the $1.5 \mathrm{hp}$ induction motor.
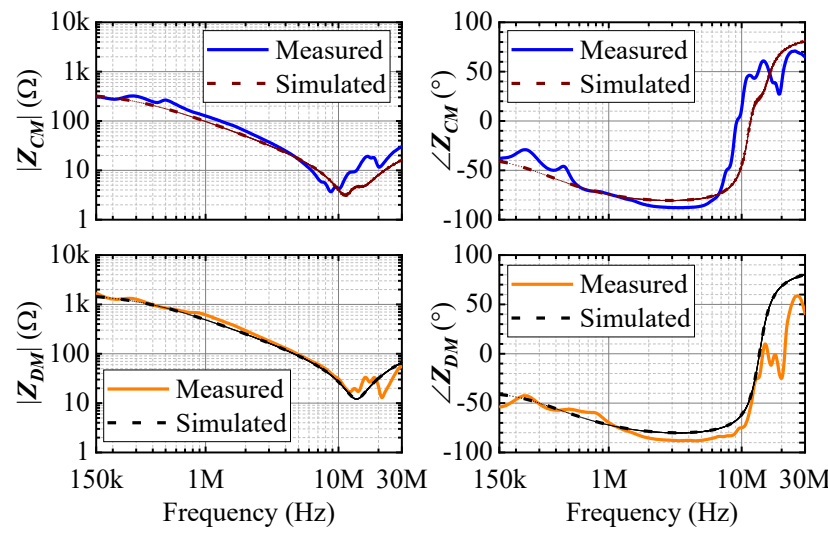

Fig. 7. Measured and simulated $\boldsymbol{Z}_{\boldsymbol{C} \boldsymbol{M}}$ and $\boldsymbol{Z}_{\boldsymbol{D} \boldsymbol{M}}$ of the $7.5 \mathrm{hp}$ induction motor.

[2] D. Han et al., "Comparative analysis on conducted CM EMI emission of motor drives: WBG versus Si devices," IEEE Trans. Ind. Electron., vol. 64, no. 10, pp. 8353-8363, Oct. 2017.

[3] J. Sun and L. Xing, "Parameterization of three-phase electric machine models for EMI simulation," IEEE Trans. Power Electron., vol. 29, no. 1, pp. 36-41, Jan. 2014.

[4] Specification for radio disturbance and immunity measuring apparatus and methods - Part 1: Radio disturbance and immunity measuring apparatus, CISPR 16-1, 1999.

[5] Information technology equipment - Radio disturbance characteristics - Limits and methods of measurement, EN 55022, 2010.

[6] Electromagnetic compatibility (EMC) - Part 6-3: Generic standardsEmission standard for equipment in residential environments, EN 610006-3, 2021.

[7] M.S. Toulabi et al., "A universal high-frequency induction machine model and characterization method for arbitrary stator winding connections," IEEE Trans. Energy Convers., vol. 34, no. 3, pp. 1164-1177, Sep. 2019.

[8] G. Suresh et al., "Predicting the transient effects of PWM voltage waveform on the stator windings of random wound induction motors," IEEE Trans. Power Electron., vol. 14, no. 1, pp. 23-30, Jan. 1999.

[9] O. Magdun, A. Binder, and C. Purcarea, "High-frequency induction machine models for calculation and prediction of common mode stator ground currents in electric drive systems," in Proc. Eur. Conf. Power Electron. Appl., 2009, pp. 1-8.

[10] H. D. Gersem and A. Muetze, "Finite-element supported transmission line models for calculating high-frequency effects in machine windings," IEEE Trans. Magn., vol. 48, no. 2, pp. 787-790, Feb. 2012.

[11] A. F. Moreira et al., "High-frequency modeling for cable and induction motor overvoltage studies in long cable drives," IEEE Trans. Ind. Appl., vol. 38, no. 5, pp. 1297-1306, Sep./Oct. 2002.

[12] B. Mirafzal, G. Skibinski, and R. Tallam, "Determination of parameters in the universal induction motor model," IEEE Trans. Ind. Appl., vol. 45, no. 1, pp. 142-151, Jan./Feb. 2009.

[13] Y. W. N. Idir et al., "High-frequency behavior models of AC motors," IEEE Trans.Magn., vol. 45, no. 1, pp. 133-138, Jan. 2009.

[14] M. Degano et al., "HF induction motor modeling using automated experimental impedance measurement matching," IEEE Trans. Ind. Electron., vol. 59, no. 10, pp. 3789-3796, Oct. 2012. 